\title{
Insertion sequence evolutionary patterns highlight convergent genetic inactivations and recent genomic island acquisitions among epidemic Burkholderia cenocepacia
}

Correspondence
Benoit Cournoyer
benoit.cournoyer@univ-lyon 1.fr

Received 3 August 2011 Accepted 29 August 2011

\author{
Arnault Graindorge, ${ }^{1,2} \dagger$ Aymeric Menard, ${ }^{1,2} \ddagger$ Claire Monnez ${ }^{1,2}$ \\ and Benoit Cournoyer ${ }^{1,2,3}$
}
${ }^{1}$ Research Group on Bacterial Opportunistic Pathogens and Environment, Université de Lyon, UMR5557 Ecologie Microbienne, Université Lyon 1, CNRS, Lyon, France
${ }^{2}$ VetAgro Sup Veterinary School, Lyon, France
${ }^{3}$ Environmental Microbiology Lyon, Biological Resource Center, Université de Lyon, UMR5557 Ecologie Microbienne, Université Lyon 1, CNRS, Lyon, France

The Burkholderia cenocepacia B\&B clone was found previously to be responsible for an epidemic outbreak within an intensive care unit in France. This clone belongs to the ST32 clonal complex, which is one of the most prevalent among French cystic fibrosis patients and is known to be related to the highly virulent ET12 clonal complex. Genomic repartition biases of insertion sequences (ISs) were investigated to improve our understanding of the evolutionary events leading to $B$. cenocepacia diversification and the emergence of such epidemic lineages. IS were used for tracking convergent genetic inactivations and recent DNA acquisitions. $B$. cenocepacia IS families and subgroups were compared in terms of genetic diversity and genomic architecture using fully sequenced genomes, PCR screening and DNA blot analysis. These analyses revealed several features shared by the B\&B and ET12 epidemic clones. IS elements showed a frequent localization on genomic islands (GI) and indicated convergent evolution towards inactivation of certain loci. The IS407 subgroup of the IS3 family was identified as a good indicator of recently acquired Gls in clone ET12. Several IS407 elements showed strain-specific or clonal complex-specific localizations. IS407 DNA probing of a DNA library built from the $B$. cenocepacia $B \& B$ epidemic clone led to the identification of a recently acquired IS407-tagged GI likely to be conjugative and integrative. The B\&B clone showed significant differences in its IS architecture from that of ST32 strains isolated from Czech cystic fibrosis patients.
†Present address: Department of Microbiology and Molecular Medicine, Faculty of Medicine, University of Geneva CMU, 1 rue Michel-Servet, 1211 Geneva 4, Switzerland.

¥Present address: IUT de Saint Brieuc, 18 rue Henri Wallon, BP 406, 22004 Saint Brieuc Cedex 1, France.

Abbreviations: Bcc, Burkholderia cepacia complex; CDS, coding sequence; CF, cystic fibrosis; DR, direct repeat; Gl, genomic island; ICE, integrative conjugative element; IS, insertion sequence; IR, inverted repeat; RGP, regions of genomic plasticity.

The GenBank/EMBL/DDBJ accession number for the sequences determined in this study are HQ248111-HQ248119, HQ438284, $\mathrm{HO} 438285$ and HQ248135-HO248167.

Four supplementary tables are available with the online version of this paper.

\section{INTRODUCTION}

Insertion sequences (ISs) are short DNA sequences that act as simple transposable elements. They encode all the genetic information required for their mobility, including a transposase coding sequence (CDS) as well as direct repeats (DR) and inverted repeats (IR) DNA that play a role in the transposition process (Mahillon \& Chandler, 1998; see http://www-is.biotoul.fr/ for a general view on IS classification issues). ISs have been described as the 'kiss of death' for a bacterial genome because of their ability to disrupt or alter gene DNA sequences and affect bacterial fitness (Wagner, 2009). This conclusion was based, in part, on the observation of a very low genetic diversity among ISs of the same family subgroup and in the same bacterial genome (Wagner, 2006). The conservation of IS elements within a bacterial species or clone appears to be dependent on the balance between the benefits and the deleterious 
effects (costs) they can have on the success of the IScolonized bacterial species or clone, as well as the ability of the organism to delete or control the expansion of these elements. A good illustration of this interplay is the successful IS evolutionary scenario that was inferred recently from Burkholderia mallei comparative genomic analyses, in which the IS under study was found to be related to the expansion of at least three IS subgroups (Song et al., 2010). B. mallei is the causative agent of glanders and has emerged from Burkholderia pseudomallei, a primary pathogen causing melioidosis (Godoy et al., 2003). IS expansion was found to match the emergence of $B$. mallei from $B$. pseudomallei. Comparative analyses of $B$. mallei genomes of several strains showed genome size reduction events, i.e. deletions and rearrangements, triggered by the amplified IS elements, predominantly of the IS407A subgroup (Song et al., 2010). IS elements can thus play a key part in the early stage of bacterial adaptation towards a specialized niche including human and animal host environments.

Among the members of the genus Burkholderia, another group of bacterial species is well known for interacting with higher organisms. This group is named the Burkholderia cepacia complex $(\mathrm{Bcc})$ and is made up of about 17 closely related species (Vanlaere et al., 2009). Species of this group are able to colonize several habitats, such as plant rhizospheres, fresh water and polluted soils, and are opportunistic pathogens that infect immunocompromised people and individuals with cystic fibrosis (CF) (Vial et al., 2011). Burkholderia cenocepacia has been identified as the most damaging Bcc species recovered from CF patients and was found to provoke a necrotic pneumonia that has been named cepacia syndrome (Saiman \& Siegel, 2004). According to multilocus restriction typing datasets, $B$. cenocepacia was found to have a panmictic population genetic structure showing the emergence of clonal epidemic lineages (Coenye \& LiPuma, 2003), which could, in the long run, become sexually isolated, like $B$. mallei from its $B$. pseudomallei parental lineage.

Several epidemic clones of $B$. cenocepacia have been described from the mid-1980s. One of the most damaging clones, ET12, was found to be distributed in North America and the UK, and has been shown to be highly transmissible (Isles et al., 1984; Johnson et al., 1994). Other North American epidemic clones have been described and named PDHC and Midwest (Coenye et al., 2004; LiPuma et al., 1988). The B\&B clone is a French epidemic clone that was described recently (Graindorge et al., 2010). This clone and its related strains are part of the ST32 clonal complex, which has been found to be involved in nosocomial and CF pulmonary tract infections and has disseminated among several occidental countries. Strains of this clonal complex have been reported as the most prevalent among French CF cohorts (Abalain et al., 2009) and as being involved in an epidemic outbreak in a Czech Republic CF cohort (Drevinek \& Mahenthiralingam, 2010). A concordance between the presence of a particular IS element and the emergence of a $B$. cenocepacia epidemic clone has been reported previously. In fact, a hybrid element containing a copy of IS1356 inserted into IS402 (later named ISBcen20) was found to be specific to the B. cenocepacia ET12 lineage (Tyler et al., 1996). Furthermore, a higher number of IS1363 (later named ISBcen2) was found among the B. cenocepacia PHDC clone than the ET12 clone (Liu et al., 2003). Kenna et al. (2006) also showed IS407 to be high in copy number among ET12 strains but absent from the PHDC clone. A link between ISs and emerging epidemic clones can thus be hypothesized from these preliminary datasets. Interestingly, B. cenocepacia was shown to harbour IS407, which in previous reports was found to trigger genome-reductive evolution in B. mallei (Song et al., 2010).

In this work, a comparative analysis of the IS architecture of the $B$. cenocepacia genome was performed to evaluate the possible use of IS elements as indicators of genomic events that could have led to the emergence of epidemic clones. These comparative datasets were then used to build a strategy to characterize some of these genomic events among the $\mathrm{B} \& \mathrm{~B}$ clone. IS elements could be trademarks of epidemic lineages and of their natural history. The genome of $B$. cenocepacia strain J2315 of the ET12 clonal complex was used as a reference to investigate these issues. A reevaluation of the IS annotations of this genome was performed to refine their allocation to particular families and subgroups. A reinvestigation of $B$. cenocepacia strain J2315 genomic islands (GIs) was also performed. These IS and GI structures were then compared with those of other strains or genomes through bioinformatics and experimental analyses. The nature of the IS-inactivated gene families, their DNA integration sites, the $\mathrm{G}+\mathrm{C}$ bias of the IS-targeted DNA sequences, the stability of the integration events (IS and GI) and their prevalence among particular clones or clonal complexes were investigated. The ET12 and $\mathrm{B} \& \mathrm{~B}$ clones showed common trends in their IS and GI architectures. These results led to the use of IS 407 as a DNA probe to detect newly acquired GIs among the $\mathrm{B} \& \mathrm{~B}$ clone.

\section{METHODS}

Burkholderia strains and PCR screenings. The Burkholderia strains or genomes used in this study are listed in Table 1. Bacteria were grown in Luria-Bertani (LB) broth at $30{ }^{\circ} \mathrm{C}$ for $24-48 \mathrm{~h}$ (Bertani, 1951). DNA extraction was performed according to the method of Pitcher et al. (1989) and the DNA was resuspended in ultrapure water.

PCR (in a volume of 25-50 $\mu \mathrm{l}$ ) was carried out using Taq DNA polymerase (Invitrogen) according to the manufacturer's guidelines except that $5 \%$ DMSO, instead of $1 \% \mathrm{~W}-1$ detergent, and $50 \mathrm{ng}$ total bacterial DNA was used. The PCR primers used in this study are described in Supplementary Table S1 (available in JMM Online). PCR cycling conditions were as follows: initial denaturation at $95{ }^{\circ} \mathrm{C}$ for $5 \mathrm{~min}$; followed by 35 cycles of $95{ }^{\circ} \mathrm{C}$ for $30 \mathrm{~s}$, annealing at the temperature given in Supplementary Table S1 for $45 \mathrm{~s}$ and DNA elongation at $72{ }^{\circ} \mathrm{C}$ for $60 \mathrm{~s}$; with a final elongation step at $72{ }^{\circ} \mathrm{C}$ for 5 min. PCR products were visualized as described by Cournoyer \& Normand (1994). PCR products were either sequenced directly or after cloning in pGEM-T Easy (Promega) using Escherichia coli DH5 $\alpha$. 
Table 1. IS elements among Burkholderia genomes

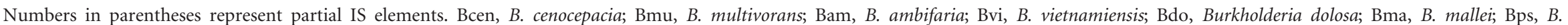
pseudomallei; Bxe, B. xenovorans; Bth, B. thailandensis.

\begin{tabular}{|c|c|c|c|c|c|c|c|c|c|c|c|c|c|c|c|}
\hline \multirow{3}{*}{$\begin{array}{l}\text { IS } \\
\text { Family }\end{array}$} & \multirow[b]{3}{*}{ Subgroup } & \multicolumn{6}{|c|}{ Bcen ${ }^{*}$} & \multirow{3}{*}{$\begin{array}{c}\text { Bmu } \\
\text { ATCC } \\
17616\end{array}$} & \multirow{3}{*}{$\frac{\text { Bam }}{\text { AMMD }}$} & \multirow{3}{*}{$\frac{\text { Bvi }}{\text { G4 }}$} & \multirow{3}{*}{$\frac{\text { Bdo }}{\text { AU0158 }}$} & \multirow{3}{*}{$\begin{array}{c}\text { Bma } \\
\text { ATCC } \\
23344\end{array}$} & \multirow{3}{*}{$\frac{\text { Bps }}{\text { K96243 }}$} & \multirow{3}{*}{$\frac{\text { Bxe }}{\text { LB400 }}$} & \multirow{3}{*}{$\begin{array}{c}\text { Bth } \\
\text { E264 }\end{array}$} \\
\hline & & \multirow{2}{*}{$\frac{\mathrm{ET} 12}{\mathrm{~J} 2315}$} & \multirow{2}{*}{$\frac{\text { B\&B }}{\text { Bpoe717† }}$} & \multicolumn{2}{|c|}{ PHDC } & \multicolumn{2}{|c|}{ Midwest } & & & & & & & & \\
\hline & & & & AU1054 & HI2424 & PC184 & MCO-3 & & & & & & & & \\
\hline \multirow[t]{23}{*}{ IS 3} & ISBcen6 & (2) & $\geqslant 1$ & & & & & & & & & & & & \\
\hline & IS Bcen7 & 2 & & & & & & & & & & & & & \\
\hline & ISBcen 9 & 9 & $\geqslant 5$ & & & & & & & & & & & & \\
\hline & ISBcen15 & 3 & & & & 1 & & & & & & & & & \\
\hline & ISBcen16 & 1 & & & & & & & 5 & & & & & & \\
\hline & ISBcen17 & 1 & & & & & & & & & & & & & \\
\hline & ISBcen21 & & & & & $1(+2)$ & 3 & & & & & & & & \\
\hline & ISBcen22 & & & 1 & 1 & 1 & 1 & & & & & & & & \\
\hline & ISBcen 23 & & & 2 & 2 & & & & & & & & & 2 & \\
\hline & IS Bp1 & & & & & & & & & & & & & & \\
\hline & IS 1416 & & & & & & & & & & & & (2) & & \\
\hline & IS 401 & & & & & & & 1 & & & & & & & \\
\hline & IS407 & $13(+1)$ & $\geqslant 6$ & & & & & 3 & & $1(+1)$ & & 91 & $3(+1)$ & & \\
\hline & ISBcen10 & 4 & & & & & & 9 & & & & & & & \\
\hline & ISButh1 & & & 1 & 2 & & & & & $1(+1)$ & & & & & 1 \\
\hline & ISButh2 & & & & & & & & & & & & & & 6 \\
\hline & IS Bxe1 & & & & & & & & & & & & & 7 & \\
\hline & ISBxe2 & & & & & & & & & & & & & 1 & \\
\hline & ISBph1 & & & & & & & & & & & & & 1 & \\
\hline & ISBmu6 & & & & & & & 4 & & & & & & & \\
\hline & ISBmu11 & & & & & 2 & & 2 & & 2 & & & & & \\
\hline & ISBvi3 & & & & & & & & & 1 & & & & & \\
\hline & ISBvi4 & & & & & & & & & 5 & & & & & \\
\hline IS 4 & ISBvil & & & & & & & & & 5 & & & & & \\
\hline \multirow[t]{2}{*}{ IS 5} & ISBcen20 & 9 & $\geqslant 8$ & & & & & 3 & (1) & 11 & & & & & \\
\hline & ISBmu2 & & & & & & & $10(+1)$ & & 3 & & & & & \\
\hline \multirow[t]{5}{*}{ IS 21} & ISBcen 28 & & & & 1 & 3 & & & & (1) & & & & & \\
\hline & IS408 & 1 & & & & & & $3(+1)$ & & 4 & & & & & \\
\hline & ISBmu1 & & & & & & & 6 & & & & & & & \\
\hline & ISBmu3 & & & & & & & 2 & & & & & $1(+1)$ & (1) & \\
\hline & ISBcen13 & 5 & & & & & & & & 1 & & & & & \\
\hline \multirow[t]{2}{*}{ IS66 } & ISBcen19 & 2 & & & & & 1 & & & & & & & & \\
\hline & ISBcen 14 & 4 & & & & & 1 & & & & & & & & \\
\hline
\end{tabular}


Table 1. cont.

\begin{tabular}{|c|c|c|c|c|c|c|c|c|c|c|c|c|c|c|c|}
\hline \multirow{3}{*}{$\begin{array}{l}\text { IS } \\
\text { Family }\end{array}$} & \multirow[b]{3}{*}{ Subgroup } & \multicolumn{6}{|c|}{ Bcen $^{*}$} & \multirow{3}{*}{$\begin{array}{c}\text { Bmu } \\
\text { ATCC } \\
17616\end{array}$} & \multirow{3}{*}{$\frac{\text { Bam }}{\text { AMMD }}$} & \multirow{3}{*}{$\frac{\text { Bvi }}{\text { G4 }}$} & \multirow{3}{*}{$\frac{\text { Bdo }}{\text { AU0158 }}$} & \multirow{3}{*}{$\begin{array}{l}\text { Bma } \\
\text { ATCC } \\
23344\end{array}$} & \multirow{3}{*}{$\frac{\text { Bps }}{\text { K96243 }}$} & \multirow{3}{*}{$\frac{\text { Bxe }}{\text { LB400 }}$} & \multirow{3}{*}{$\frac{\text { Bth }}{\text { E264 }}$} \\
\hline & & \multirow{2}{*}{$\frac{\mathrm{ET} 12}{\mathrm{~J} 2315}$} & \multirow{2}{*}{$\frac{\mathrm{B} \& \mathrm{~B}}{\mathrm{Bpoe} 717 \dagger}$} & \multicolumn{2}{|c|}{ PHDC } & \multicolumn{2}{|c|}{ Midwest } & & & & & & & & \\
\hline & & & & AU1054 & HI2424 & PC184 & MCO-3 & & & & & & & & \\
\hline \multirow[t]{9}{*}{ IS 110} & ISBcen 1 & 1 & & & & (1) & & & & & & & & & \\
\hline & ISBcen 2 & 1 & 0 & 5 & 5 & & & & & & & & & & \\
\hline & ISBcen 3 & 2 & & & & & & & & & & & & & \\
\hline & İsBcen 4 & $1(+1)$ & $\geqslant 3$ & & & & & & & & & & & (1) & \\
\hline & İSBcen 5 & 3 & & & & & & & & & & & & & \\
\hline & ISBfun 1 & & & & & & & & & & & & & 1 & \\
\hline & ISBcen 8 & 12 & $\geqslant 7$ & 2 & 1 & 3 & 4 & 4 & & & & & & & \\
\hline & IS Bma3 & & & 8 & 3 & & & 2 & & 3 & & & $1(+1)$ & $3(+1)$ & 5 \\
\hline & ISBfun2 & & & & & & & & & & & & & 1 & \\
\hline \multirow[t]{7}{*}{ IS 256} & IS 1356 & 2 & & 4 & 2 & & 3 & 7 & 1 & 3 & 4 & & & (1) & $6(+1)$ \\
\hline & IS 1413 & & & & & & & & & & & & & 2 & \\
\hline & ISBcen 18 & 1 & & & & 3 & 3 & & & 8 & 3 & & (1) & & \\
\hline & IS 931 & & & & & & & & & 7 & & & & & \\
\hline & IS 1490 & & & & & & & & & 1 & & & & & \\
\hline & IS 406 & & & & & & & 6 & & & & & & & \\
\hline & ISRso7 & & & 2 & 2 & 1 & & & & & & & & & \\
\hline \multirow[t]{4}{*}{ IS 481} & ISBcen 25 & & & 2 & 2 & & & & & & & & & & \\
\hline & ISBcen 26 & & & 1 & 1 & & & & & & & & & & \\
\hline & IS $B x e 3$ & & & & & & & & & & & & & 5 & \\
\hline & ISBxe4 & & & & & & & & & & & & & 3 & \\
\hline IS $L 3$ & IS Bmal & & & & & 2 & & & & 1 & & $17(+3)$ & 6 & & 7 \\
\hline \multirow[t]{4}{*}{ Others } & IS Bma2 & & & & & & & & & & & 46 & 5 & (2) & \\
\hline & ISBfun4 & & & & & & & & & & & & & 1 & \\
\hline & IS 1071 & & & & & & & (1) & & & & & & 2 & \\
\hline & Unknown & 3 & & 6 & 4 & & & & & 14 & 1 & & & & \\
\hline Total & & 84 & $\geqslant 31$ & 34 & 26 & 20 & 16 & 65 & 7 & 74 & 8 & 157 & 22 & 35 & 26 \\
\hline
\end{tabular}

*The genome of Burkholderia lata strain ATCC 17760 (also named 383) showed no IS.

$\dagger$ IS elements among the B\&B clone were detected by DNA blot analyses; the $\geqslant$ sign indicates that DNA hybridization may have underestimated the number of copies. 
pGEM-T was extracted using a Qiagen Plasmid Plus Midi kit (Qiagen). DNA sequencing of the PCR products and pGEM-T plasmids were performed by Cogenics (Grenoble, France).

All DNA sequences obtained in this work were deposited in GenBank and are listed in Supplementary Table S2.

B\&B clone genomic library and DNA blot analysis. DNA blot analyses were performed as follows. Genomic DNA $(5 \mu \mathrm{g})$ was digested with $1 \mathrm{U}$ EcoRI (Fermentas) $\mu \mathrm{g}^{-1}$ for $16 \mathrm{~h}$ at $37^{\circ} \mathrm{C}$. The digested DNA fragments were separated by electrophoresis using a $1 \%$ agarose gel, for $6 \mathrm{~h}$ at $100 \mathrm{~V}$. DNA blots were carried out using Genescreen nylon membranes and following the procedure described by the manufacturer. DNA probes were labelled using a random priming labelling kit (Amersham-Pharmacia). After hybridization, the membrane was washed at $65{ }^{\circ} \mathrm{C}$. Autoradiography was performed according to the method of Sambrook et al. (1989).

The bpoe717 B\&B strain of B. cenocepacia was used to build a DNA library. This strain was isolated in 2004 from a mechanically ventilated patient hospitalized in a French intensive-care unit who died of respiratory failure 3 days after the diagnosis of a nosocomial pulmonary infection (Graindorge et al., 2010). Its genomic DNA was extracted as described by Pitcher et al. (1989) and partially digested by Sau3AI (Fermentas). The digested DNA fragments were separated by sucrose density-gradient centrifugation. Fragments in the range of $30 \mathrm{~kb}$ were ligated into pCPP47 as described by Bauer \& Collmer (1997). Ligation was performed using a $1: 1$ ratio of vector and insert. Lambda packaging of ligated DNA and delivery of encapsidated cosmids into $E$. coli were performed as described by Menard et al. (2007). Transductants were plated on LB agar supplemented with tetracycline $\left(10 \mu \mathrm{g} \mathrm{ml}^{-1}\right)$ and ampicillin $(100 \mu \mathrm{g}$ $\mathrm{ml}^{-1}$ ) and incubated overnight at $37^{\circ} \mathrm{C}$. In total, 1248 transductants were transferred into 96-well plates containing LB medium supplemented with tetracycline $\left(12.5 \mu \mathrm{g} \mathrm{ml} \mathrm{m}^{-1}\right)$, and were also transferred onto large LB agar plates supplemented with tetracycline $\left(12.5 \mu \mathrm{g} \mathrm{ml}^{-1}\right)$ and ampicillin $\left(30 \mu \mathrm{g} \mathrm{ml}^{-1}\right)$ to estimate the proportion of empty pCPP 47 vectors. Only $8.3 \%$ of the plasmids of the gene library were found to be false positives. The gene ecf $B$, known to be present in a single copy in the B\&B genome, was used to determine the number of bpoe717 genomes likely to have been cloned during the DNA library construction process. ecfB DNA probing of DNA blots of the 1248 library clones gave five positive hybridization signals, indicating successful cloning of about five bpoe717 genomes in the library. When required, plasmids of the gene library were extracted using a plasmid purification kit (Qiagen).

Bioinformatics analysis of IS and GIs. The full-genome sequence of B. cenocepacia J2315 was retrieved from the Sanger Institute website (http://www.sanger.ac.uk/resources/downloads/bacteria/burkholderiacenocepacia.html). B. cenocepacia AU1054, B. cenocepacia HI2424, Burkholderia ambifaria AMMD, Burkholderia sp. 383, Burkholderia vietnamiensis G4, Burkholderia multivorans ATCC 17616 and Burkholderia xenovorans LB400 genome sequences were retrieved from the Joint Genome Institute website (http://genome.jgi-psf.org). B. mallei ATCC 23344, B. pseudomallei K96243 and Burkholderia thailandensis E264 genome sequences were retrieved from GenBank. The $B$. cenocepacia PC184 genome was retrieved from the Broad Institute (http://www.broadinstitute.org/annotation/genome/burkholderia_ cenocepacia/).

Genomic analyses of IS architectures were performed using a local BLAST approach allowing comparison of IS sequences retrieved from B. cenocepacia genomes, the IS databases (http://www-is.biotoul.fr/) and the above genome sequences. BLAST analyses were performed using the latest executables (ftp://ftp.ncbi.nih.gov/blast/executables/
LATEST). The Artemis Comparison Tool (ACT) software was used to visualize graphically some of the BLAST results (Carver et al., 2005). Similarly, pairwise B. cenocepacia genome comparisons were performed by BLASTN and visualized by the ACT graphic tool.

Molecular phylogeny analyses. Multiple alignments of DNA sequences were computed using CLUSTAL W. The distance between sequence pairs, the inferred phylogenetic trees and bootstrap values were all computed using the MEGA4 graphic tool (Kumar et al., 2004). Phylogenetic trees were reconstructed using the neighbour-joining method (Saitou \& Nei, 1987). A total of 1000 bootstrap replicates were performed. The alignments carried out in this study are available on the microbial ecology research centre FTP server: http://ecomicro. univ-lyon1.fr/poe/IS-phylogeny-AN-alignments.rar.

\section{RESULTS}

\section{The most prevalent $B$. cenocepacia IS families}

Fully assembled B. cenocepacia genomes of strains J2315, AU1054, HI2424 and PC184 were used to estimate IS numbers and build up a set of reference ISs for further investigations. ISs were detected by BLASTN searches and ACT analyses. These investigations were completed by DNA blot analyses of IS numbers of certain families or subgroups among the B. cenocepacia $\mathrm{B} \& \mathrm{~B}$ clone. The DNA probes used in these analyses were obtained by PCR from strain J2315 DNA, using the DNA primers indicated in Supplementary Table S1. Additional sequenced genomes of the Bcc and members of the genus Burkholderia were included in these analyses. B. cenocepacia sequenced genomes were found to harbour 16-84 IS elements, with the latter value indicative of recent bursts (Table 1). IS481 and ISL3 were only found among the PHDC genomes and the PC184 genome, respectively. IS DNA hybridization of genomic DNA blots of strain bpoe717 of the $\mathrm{B} \& \mathrm{~B}$ clone was performed to roughly estimate the copy numbers of three IS families selected according to their distribution among B. cenocepacia strains. IS3 and IS110 were selected as representatives of the most prevalent families, and IS5 was selected as a representative of those having a narrow distribution (Table 1 ). The results of these DNA hybridizations suggested a B\&B IS architecture much closer to that of the sequenced J2315 genome than those of the strains PHDC, PC184 and MCO-3.

Genomic localization of these IS elements was investigated for the available B. cenocepacia sequenced genomes (Figs 1 and 2). These analyses showed a high repartition of $>80 \%$ of the IS elements outside the CDS of the PC184 and PHDC (HI2424 and AU1054) genomes. However, $38 \%$ of J2315 IS elements were found inside CDS. ISs found among the CDSs showed a strong bias towards colonization of other IS elements, creating IS genomic islets in the J2315 genome (Fig. 2). Overall, out of 84 IS elements, 53 were localized on chromosome 1 of J2315 genome, 16 were localized on chromosome 2, 14 were localized on chromosome 3 and one was localized on its endogenous plasmid. 
(a)

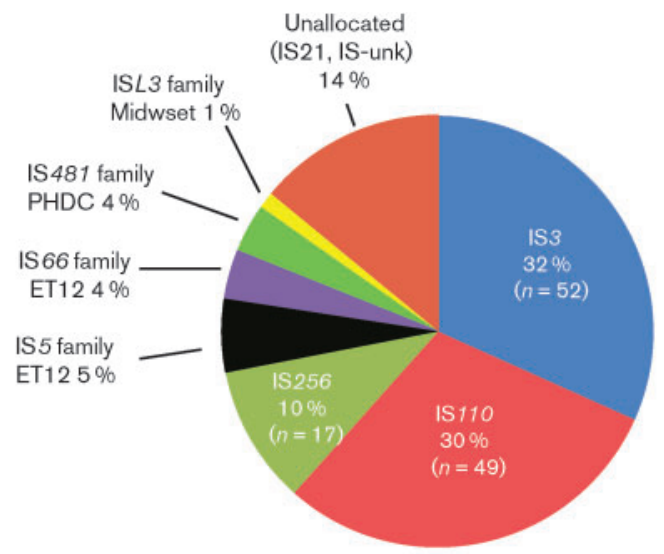

(b)

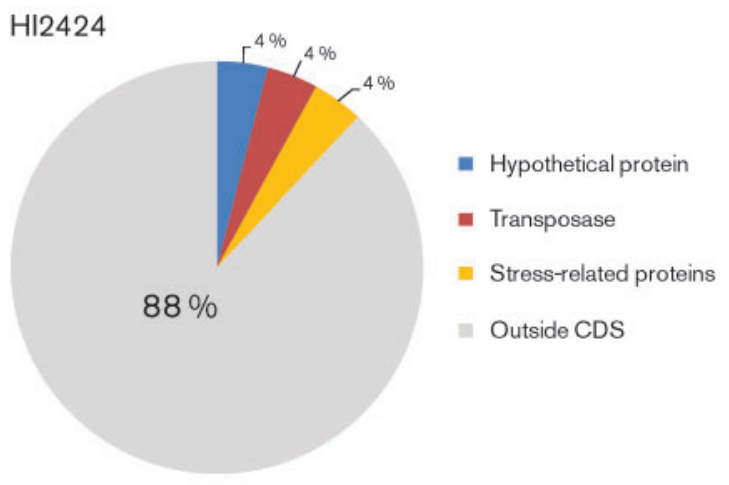

AU1054

PC184

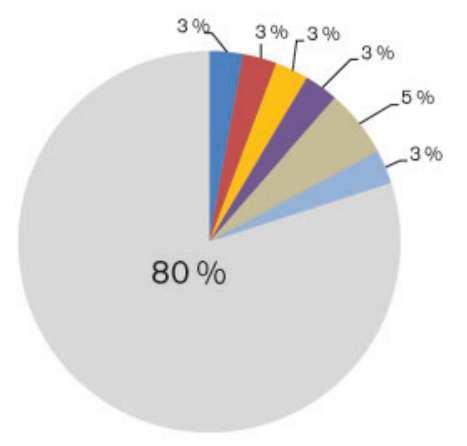

- Hypothetical protein

- Transposase

= Stress-related proteins

- Amino acid metabolism

n Transport-related proteins

m Carbohydrate metabolism

= Outside CDS

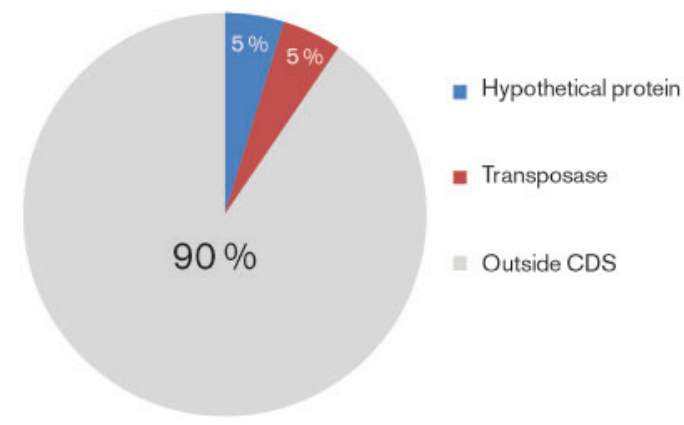

$\mathrm{J} 2315$

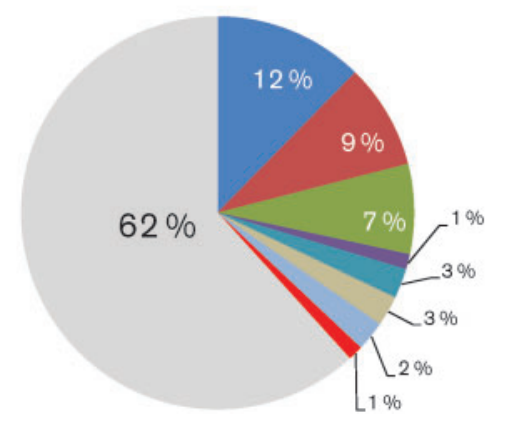

- Hypothetical protein
| Transposase
| DNA-related protein
- Amino acid metabolism
| Phage-related protein
| Transport-related protein
| Carbohydrate metabolism
- VitB6/co-factor biosynthesis
" Outside CDS

Fig. 1. Prevalence and localization of IS families among B. cenocepacia sequenced genomes. (a) Relative proportions of IS families among B. cenocepacia sequenced genomes of strains J2315, AU1054, HI2424 and PC184. (b) Classes of disrupted ORFs. The relative proportions of IS-disrupted CDSs for B. cenocepacia sequenced genomes of strains J2315, AU1054, HI2424 and PC184 are shown.

\section{IS genetic diversity and decay}

The IS3, IS110 and IS256 families showed a broad occurrence among B. cenocepacia. Most ISs (>85\%) showed a classical genetic content matching previously described elements. Other ISs showed signs of genetic decay, including mutational inactivation events and truncations. The truncated and inactivated pseudo-ISs (indicated by an ' $\mathrm{m}$ ' in front of the IS) of the J2315 genome are indicated on its chromosomal map in Fig. 2. Phylogenetic analyses were performed to determine the number of subgroups among each family (Table 1 and data not shown). Three IS subgroups (IS407, ISBcen8 and IS1356), identified as being part of the most prevalent families, were found to be distributed in at least $50 \%$ of the sequenced genomes analysed in this study (Table 1). Supplementary Tables S2 and S3 summarize the distribution and DNA sequence analysis of IS407-colonized B. cenocepacia DNA loci of distinct clonal complexes or recA PCR RFLP groups (Mahenthiralingam et al., 2000). The inactive IS407-h and IS407-i elements were, with IS407-n, the most common 

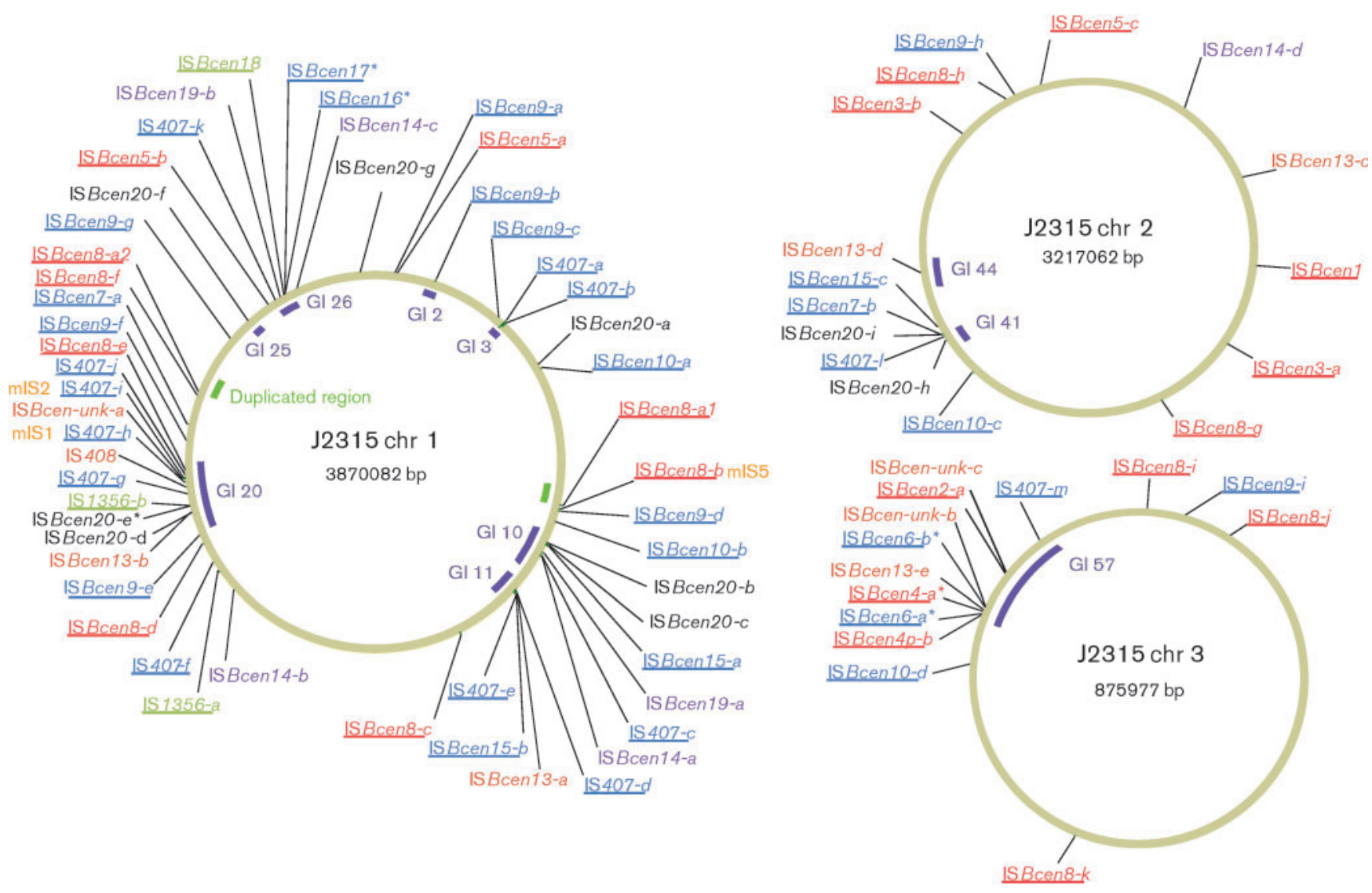

Fig. 2. Chromosomal localization of $B$. cenocepacia J2315 IS elements. IS $407-n$ is plasmid-borne and is not shown. The most prevalent IS subgroups are underlined and indicated using the colour code in Fig. 1(a). IS5 family elements are indicated in black and other narrowly distributed ISs among B. cenocepacia strains are in purple. The chromosomal positions of narrowly distributed Gls among the Bcc harbouring IS elements (see Table 3) and the positions of mutated/truncated ISs (mIS1, mIS2 and $\mathrm{mIS5}$ ) are indicated. Asterisks indicate an IS inserted inside another IS element.

elements among the panel of strains that were investigated. IS407-n was detected on the J2315 plasmid harbouring a cluster of genes encoding a type IV secretion system involved in onion tissue infection (Holden et al., 2009). Some DNA sites colonized by IS 407 among the J2315 genome were found to be inactivated by other DNA inserts in some other B. cenocepacia strains (Table 2 and Supplementary Table S3). DNA sequencing of the matching IS407-a DNA region of strains LMG 12614, H111, LMG 19240 and LMG 21461 revealed DNA segments encoding a partial C subunit of an excinuclease described in V. eiseniae EF01-2 (Supplementary Table S3). This segment is perfectly inserted at the matching DR sequence colonized by IS407-a in the J2315 genome and indicates genetic convergence towards inactivation of this locus. This locus itself is non-coding but is surrounded by CDS encoding hypothetical proteins. Similar genetic convergences towards inactivation of particular genetic loci were also observed for the IS407-m- and -1-colonized DNA sites. The matching IS407-1 DR homologous site in strain LMG 12614 was inactivated by a partial CDS encoding a putative phage tail protein (Supplementary Table S3), and the matching IS407-m DR homologous site in strain LMG 21461 was inactivated by a DNA fragment with no significant homologue in the databases.

With regard to the $\mathrm{B} \& \mathrm{~B}$ clone, 10 of the 14 loci colonized by an IS 407 in J2315 could be detected in the genome of strain bpoe717 (B\&B clone). Six of these loci showed an IS407 and four did not harbour any IS. This is the highest number of IS407-colonized DNA loci matching those of J2315 outside the ET12 clone (Table 2). The four remaining loci could not be detected. A phylogenetic tree of IS407 DNA sequences was reconstructed (Fig. 3). This tree showed B. cenocepacia IS407 elements, including those of the B\&B clone, divided into two clusters, ' $a$ ' and ' $b$ '. The ' $a$ ' cluster grouped DNA sequences that were identical (e.g. IS407-l) or almost identical to the IS407-n element, which is located on a plasmid. The ' $b$ ' cluster grouped the IS407-h and -i elements. It is noteworthy that IS407-k was found in the opposite orientation among the $\mathrm{B} \& \mathrm{~B}$ clone, and nucleotide deletions inactivating orf $A$ and $\operatorname{orf} B$ of the transposase CDS were observed (GenBank 
Table 2. PCR screening of selected IS407 sites among a panel of $B$. cenocepacia strains

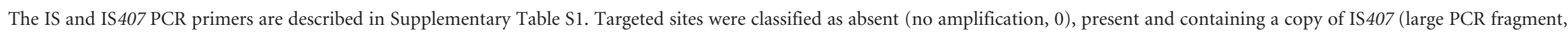

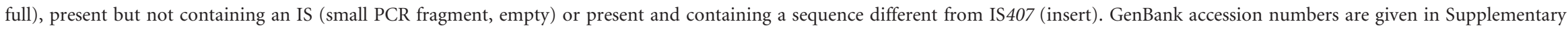
Table S2.

\begin{tabular}{|c|c|c|c|c|c|c|c|c|c|c|c|c|c|c|c|c|}
\hline \multirow[t]{3}{*}{ IS407 copy* } & \multirow[t]{3}{*}{ PCR primers } & \multicolumn{5}{|c|}{ IIIA } & \multicolumn{6}{|c|}{ IIIB } & \multirow[t]{2}{*}{ IIIC } & \multirow[t]{2}{*}{ IIID } & \multicolumn{2}{|c|}{$\begin{array}{l}\text { Expected PCR } \\
\text { products (bp) } \\
\text { from screening }\end{array}$} \\
\hline & & \multicolumn{3}{|c|}{ ET12 } & \multirow[b]{2}{*}{ B\&B clone } & \multirow[b]{2}{*}{ H111 } & \multirow[b]{2}{*}{$\begin{array}{l}\text { LMG } \\
18828\end{array}$} & \multirow[b]{2}{*}{$\begin{array}{l}\text { LMG } \\
16654\end{array}$} & \multicolumn{2}{|c|}{ PHDC } & \multicolumn{2}{|l|}{$\dagger$} & & & IS full & IS \\
\hline & & $\mathrm{J} 2315$ & $\begin{array}{l}\text { LMG } \\
12614\end{array}$ & $\begin{array}{c}\text { LMG } \\
18826\end{array}$ & & & & & HI2424† & AU1054 $\dagger$ & $\begin{array}{c}\text { LMG } \\
18829\end{array}$ & $\begin{array}{c}\text { LMG } \\
18832\end{array}$ & $\begin{array}{c}\text { LMG } \\
19240\end{array}$ & $\begin{array}{l}\text { LMG } \\
21461\end{array}$ & & \\
\hline IS $407-a$ & 407aF/407aR & Full & Insert $\neq$ & Full & 0 & Insert $\neq$ & 0 & 0 & 0 & 0 & 0 & 0 & Insert $\ddagger$ & Insert $\neq$ & 1400 & 700 \\
\hline IS $407-b$ & SI10/SI11 & Full & Empty & Full & 0 & 0 & 0 & 0 & 0 & 0 & 0 & 0 & 0 & 0 & 1585 & 349 \\
\hline$\overline{\text { IS 407-c }}$ & $407 \mathrm{cF} / 407 \mathrm{cR}$ & Full & Empty & $\begin{array}{l}\text { Full } \neq \\
(100)\end{array}$ & $\begin{array}{c}\text { Empty } \ddagger \\
(100)\end{array}$ & Empty & Empty & Empty & 0 & 0 & 0 & 0 & 0 & $\begin{array}{c}\text { Empty } \neq \\
(100)\end{array}$ & 1802 & 600 \\
\hline IS407-d & SI8/SI17 & Full & Empty & Full & $\begin{array}{c}\text { Empty } \neq \\
(98)\end{array}$ & 0 & 0 & 0 & 0 & 0 & 0 & 0 & $\begin{array}{c}\text { Empty } \ddagger \\
(100)\end{array}$ & $\begin{array}{c}\text { Empty } \neq \\
(98)\end{array}$ & 1439 & 383 \\
\hline IS407-e & $407 \mathrm{eF} / 407 \mathrm{eR}$ & Full & $\begin{array}{c}\text { Empty } \neq \\
(100)\end{array}$ & $\begin{array}{l}\text { Full } \neq \\
(100)\end{array}$ & 0 & 0 & 0 & 0 & 0 & 0 & 0 & 0 & 0 & $\begin{array}{c}\text { Empty } \neq \\
(99)\end{array}$ & 1411 & 385 \\
\hline IS407-f & $407 \mathrm{fF} / 407 \mathrm{fR}$ & Full & Empty & Full & $\begin{array}{c}\text { Empty } \neq \\
(97)\end{array}$ & Empty & Empty & Empty & Empty & Empty & Empty & 0 & 0 & $\begin{array}{c}\text { Empty } \neq \\
(90)\end{array}$ & 1715 & 475 \\
\hline$\underline{\text { IS } 407-g}$ & SI21/SI22 & Full & $\begin{array}{c}\text { Empty } \ddagger \\
(100)\end{array}$ & Full & 0 & 0 & 0 & 0 & 0 & 0 & 0 & 0 & 0 & 0 & 1557 & 321 \\
\hline IS $407 h$ & SI23/SI24 & Full & Full & Full & Full $¥$ (99) & 0 & 0 & 0 & 0 & 0 & 0 & 0 & 0 & Full & 1430 & 193 \\
\hline IS $407-i$ & 407iF/407iR & Full & $\begin{array}{l}\text { Full } \ddagger \\
(100)\end{array}$ & Full & Full $\ddagger(99)$ & Full & 0 & $\begin{array}{l}\text { Full } \ddagger \\
(100)\end{array}$ & 0 & 0 & 0 & Full & Full $\ddagger(99)$ & Full $\ddagger(99)$ & 1000 & 470 \\
\hline$\underline{\text { IS } 407-j}$ & 407jF/407jR & Full & $\begin{array}{c}\text { Empty } \ddagger \\
(100)\end{array}$ & $\begin{array}{l}\text { Full } \neq \\
(100)\end{array}$ & Empty & Empty & 0 & $\begin{array}{c}\text { Empty } \ddagger \\
(100)\end{array}$ & 0 & 0 & 0 & 0 & $\begin{array}{c}\text { Empty } \ddagger \\
(100)\end{array}$ & $\begin{array}{c}\text { Empty } \neq \\
(100)\end{array}$ & 1555 & 380 \\
\hline IS407-k & SI5/SI7 & Full & $\begin{array}{c}\text { Empty } \neq \\
(100)\end{array}$ & $\begin{array}{l}\text { Full } \neq \\
(100)\end{array}$ & Full $¥(89)$ & 0 & 0 & 0 & 0 & 0 & 0 & 0 & 0 & $\begin{array}{c}\text { Empty } \neq \\
(100)\end{array}$ & 1470 & 444 \\
\hline IS407-l & SI27/SI20 & Full & Insert $\ddagger$ & Full & $\begin{array}{l}\text { Full\$ } \\
(100)\end{array}$ & 0 & 0 & 0 & 0 & 0 & 0 & 0 & 0 & 0 & 1408 & 382 \\
\hline IS $407-m$ & SI3/SI2 & Full & Empty & Full & Full $\ddagger(56)$ & 0 & 0 & 0 & 0 & 0 & 0 & 0 & Full & Insertł & 1437 & 201 \\
\hline IS $407-n$ & SI14/SI15 & Full & $\begin{array}{l}\text { Full } \ddagger \\
(100)\end{array}$ & $\begin{array}{l}\text { Full } \neq \\
(100)\end{array}$ & $\begin{array}{c}\text { Full } \neq \\
(100)\end{array}$ & 0 & 0 & 0 & 0 & 0 & 0 & 0 & $\begin{array}{l}\text { Full } \neq \\
(100)\end{array}$ & $\begin{array}{l}\text { Full } \neq \\
(100)\end{array}$ & 1612 & 376 \\
\hline
\end{tabular}




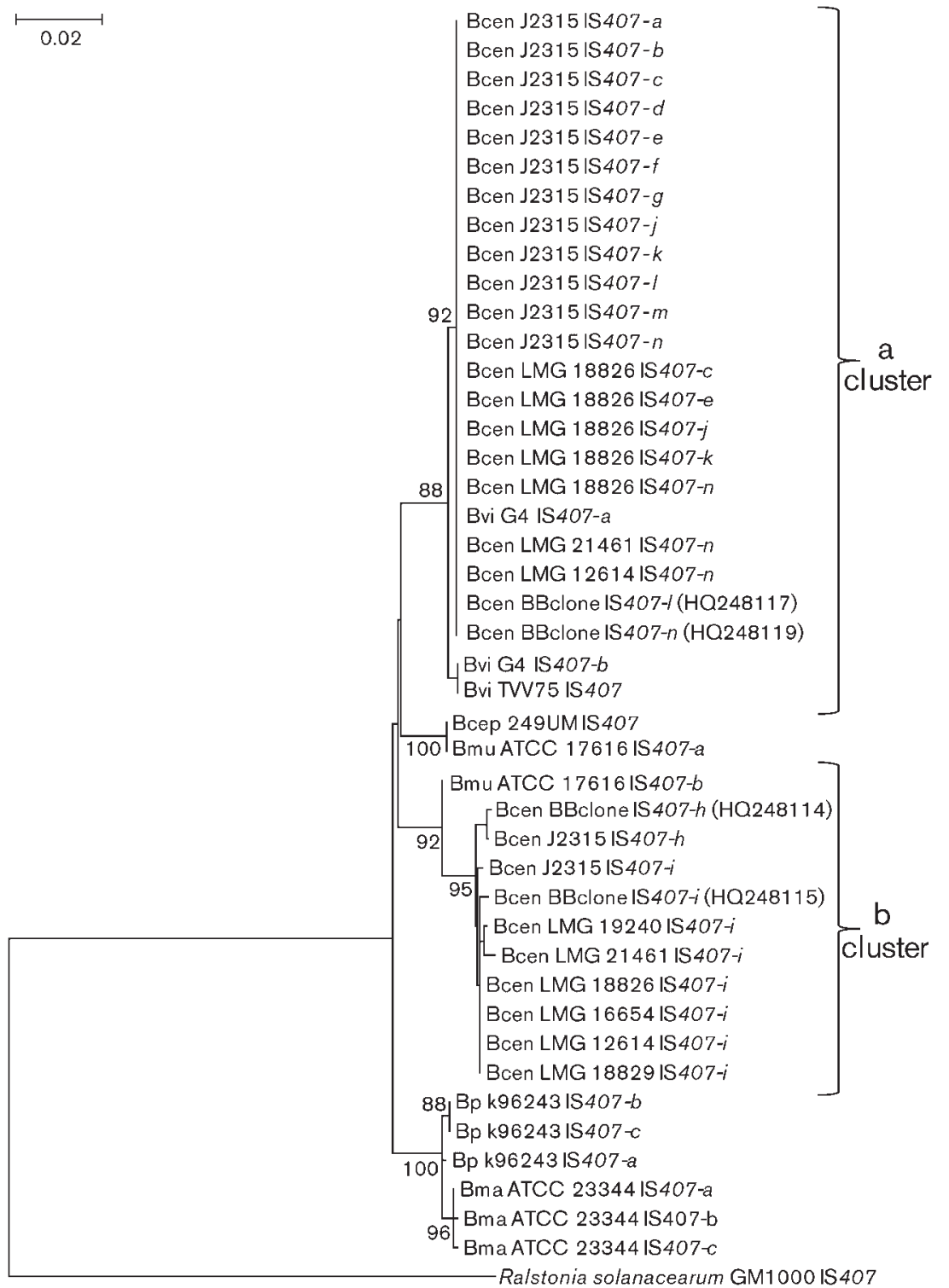

Fig. 3. Neighbour-joining phylogenetic tree of IS407 transposase DNA sequences. DNA sequences were retrieved from sequenced Burkholderia genomes obtained by DNA sequencing of PCR products or from GenBank. A total of 1123 sites were analysed (with pairwise removal of gap-containing sites) using Kimura's two-parameter model. Bar, 0.02 substitutions per nucleotide position. Bootstrap values $>85 \%$ are indicated. Bcen, B. cenocepacia; Bvi, B. vietnamiensis; Bmu, B. multivorans; Bp, B. pseudomallei; Bcep, B. cepacia; Bma, B. mallei. accession no. HQ248116). As in the J2315 genome, this IS element disrupted a gene involved in capsule synthesis. In the case of IS407-m, a genomic rearrangement significantly modified this element within the $B \& B$ clone but it was found to be in the same orientation (GenBank accession no. HQ248118). orfA and orfB were found to be partial and were located 155 bp upstream of the left IR (IR-L).

To explore the events that could explain the absence of IS407 elements among several B. cenocepacia strains, an ACT comparison between J2315 IS407-targeted DNA regions and the HI2424 sequenced genome (PHDC lineage) was performed. This analysis showed that most IS407 elements found in the J2315 genome were on DNA regions not present in the PHDC genomes (Table 2). Only IS407-f was found to be inserted in a DNA region also observed in the HI2424 genome (but without an IS407 insertion).

\section{IS and regions of genomic plasticity (RGP)}

RGP are DNA regions that appear to be missing from a genome after performing pairwise comparisons with a closely related genome and were identified through ACT comparisons of the J2315 and PHDC genomes (strains HI2424 and AU1054). Not all RGP DNA regions identified in this work matched the full criteria used to define classical GIs, such as the presence of int and tRNA genes, but the GI abbreviation was used for simplicity in order to name all these RGP regions (Table 3 and Supplementary Table S4). Overall, 1.34 Mb of J2315 DNA was found to be absent from the PHDC genomes. These DNA segments were divided into 58 DNA regions with sizes ranging from 4.1 to $142.2 \mathrm{~kb}$ and a G+C content between 48.1 and $71.4 \mathrm{~mol} \%$ (Table 3 and Supplementary Table S4). Of the 58 RGP, 31 showed a distribution among other Bcc strains by BLASTN, with partial or complete identities 


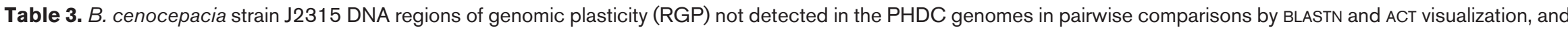
not detected in other Burkholderia spp. by BLAST analyses

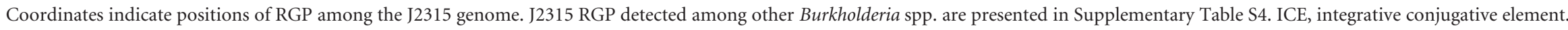

\begin{tabular}{|c|c|c|c|c|c|c|c|}
\hline \multirow[t]{2}{*}{ RGP name } & \multirow[t]{2}{*}{ Coordinates } & \multirow{2}{*}{$\begin{array}{l}\text { Size } \\
(\mathbf{k b})\end{array}$} & \multirow[t]{2}{*}{ Nature $^{*}$} & \multirow{2}{*}{$\frac{\mathrm{G}+\mathrm{C}}{(\mathrm{mol} \%)}$} & \multirow[t]{2}{*}{ IS } & \multicolumn{2}{|c|}{ Special features } \\
\hline & & & & & & Left end & Right end \\
\hline \multicolumn{8}{|c|}{ Chromosome 1} \\
\hline GI1 & $100361-125265$ & 25.00 & Prophage (BcenGI1) & 68.5 & No & tRNA-Arg & p tRNA-Arg \\
\hline GI2 & $188011-204429$ & 16.40 & Likely ICE (BcenGI2) & 55.7 & ISBcen $9-\mathrm{b}$ & tRNA-Ala & p tRNA-Ala \\
\hline GI3 & $449978-463082$ & 13.10 & Miscellaneous island (BcenGI3) & 56.5 & IS $407-a,-b$ & None & None \\
\hline GI8 & $1032360-1036537$ & 4.18 & Miscellaneous island (BcenGI4) & 42.82 & No & None & tRNA-Met \\
\hline GI10 & $1222566-1315385$ & 92.80 & Miscellaneous island (BcenGI5) & 60.3 & $\begin{array}{l}\text { ISBcen20-b,-c, ISBcen15-a, } \\
\text { ISBcen19-a, IS407-c, ISBcen14-a }\end{array}$ & Group I intron & None \\
\hline GI11 & $1402882-1437088$ & 34.20 & Miscellaneous island (BcenGI6) & 58.8 & IS407-d, ISBcen13-a, ISBcen15-b, IS407-e & tRNA-Arg & $\mathrm{p}$ tRNA-Arg \\
\hline GI13 & $1481512-1503701$ & 22.19 & Miscellaneous island & 66.55 & No & None & None \\
\hline GI14 & $1728919-1766584$ & 37.70 & Prophage KS10, $\mu$-like (BcenGI7) & 62.9 & No & BCAL1558p & BCAL1558p \\
\hline GI19 & $2656516-2666220$ & 9.71 & Putative LPS biosynthesis & 67.76 & No & None & None \\
\hline GI20 & $2738065-2859923$ & 121.90 & $\begin{array}{l}\text { Miscellaneous island, one } \\
\text { unknown integrase (BcenGI8) }\end{array}$ & 61.4 & $\begin{array}{l}\text { ISBcen13-b, ISBcen20-d, -e, IS1356-b, IS407-g, } \\
\text { IS408, IS407-h, -i, -j, ISBcen8-e, ISBcen-unk-a }\end{array}$ & None & BCAL2601p \\
\hline GI22 & $3241588-3257909$ & 16.30 & $\begin{array}{l}\text { Possible phage remnant } \\
\text { (BcenGI9) }\end{array}$ & 62.8 & No & tmRNA & None \\
\hline GI24 & $3366796-3381064$ & 14.27 & Putative metabolism (BcenGI10) & 59.4 & No & tRNA-Gly & p tRNA-Gly \\
\hline GI25 & $3411938-3424737$ & 12.80 & Glycosyl/acetyl transferase & 59.01 & ISBcen20-f & None & None \\
\hline GI26 & $3522290-3562993$ & 40.70 & Putative capsular biosynthesis & 55.54 & $\begin{array}{l}\text { IS407-k, ISBcen19-b, } \\
\text { ISBcen18, ISBcen17, ISBcen16 }\end{array}$ & None & None \\
\hline \multicolumn{8}{|c|}{ Chromosome 2} \\
\hline GI27 & 70049-84707 & 14.66 & Miscellaneous island & 55.41 & No & Partial ISBcen 20 & None \\
\hline GI28 & $168377-182238$ & 13.86 & Miscellaneous island & 59.49 & No & None & None \\
\hline GI33 & $1140183-1186975$ & 46.80 & Prophage (BcenGI12) & 63.2 & No & tRNA-SeC & $\mathrm{p}$ tRNASeC \\
\hline GI39 & 1890941-1895098 & 4.10 & Miscellaneous island & 65.78 & No & None & None \\
\hline GI40 & $2011726-2019173$ & 7.50 & Putative prophage & 66.1 & No & None & None \\
\hline GI41 & $2091701-2137922$ & 46.20 & Prophage (BcenGI13) & 54.3 & ISBcen20-h, IS407-l, ISBcen20-i & BCAM1874p & BCAM1874p \\
\hline GI43 & $2260643-2275008$ & 14.30 & Type III secretion system & 71.37 & No & None & None \\
\hline GI44 & 2332339-2395714 & 63.30 & Putative metabolism & 63.04 & ISBcen13-d & None & None \\
\hline GI46 & $2877034-2891133$ & 20.20 & Bleomycin resistance & 65.5 & No & None & None \\
\hline \multicolumn{8}{|c|}{ Chromosome 3} \\
\hline GI55 & $524936-535707$ & 10.70 & Miscellaneous island & 69.68 & No & None & None \\
\hline GI56 & 573207-609954 & 36.70 & Prophage BcepMu (BcenGI14) & 62.8 & No & None & None \\
\hline GI57 & 703084-794700 & 91.60 & $\begin{array}{l}\text { Putative metabolism. Intron } \\
\text { group II class E with two } \\
\text { unknown integrases }\end{array}$ & 61.33 & $\begin{array}{l}\text { ISBcen6-a, ISBcen4-a, ISBcen6-b, } \\
\text { ISBcen4-b, ISBcen13-e, ISBcen2-a, } \\
\text { IS407-m, ISBcen-unk-b, ISBcen-unk-c }\end{array}$ & None & None \\
\hline GI58 & $839553-856005$ & 16.40 & $\begin{array}{l}\text { Putative peptidoglycan-binding } \\
\text { membrane protein }\end{array}$ & 69.9 & No & None & None \\
\hline
\end{tabular}


(Supplementary Table S4). IS elements were detected only on three RGP (GI18, GI29 and GI50) of these broadly distributed' Bcc RGP elements (Fig. 4a). The remaining 27 RGP of the J2315 genome showed no significant identity to the Bcc sequenced genomes available at the time of this study (Table 3). Of these 'narrowly distributed' Bcc RGP, $37 \%$ showed IS elements ( 43 of the total 84 IS elements; Table 3), and six of these (GI10, GI11, GI20, GI26, GI41 and GI57) harboured $83 \%$ of the total number of ISs located on RGP (35 of the 42 ISs located on RGP) (Fig. 4b). Thirteen of these RGP have been described previously by Holden et al. (2009). The highest number of IS elements was detected among low $\mathrm{G}+\mathrm{C}$ content RGP or GIs, and no IS was detected among RGP with a DNA G + C content similar to or higher than that of the overall genome (Fig. 4c). RGP harbouring a high number of IS elements showed sizes ranging from 34 (GI11) to $122 \mathrm{~kb}$ (GI20) and DNA $\mathrm{G}+\mathrm{C}$ contents of 54 (GI41) to $61 \mathrm{~mol} \%$ (GI20 and GI57). These narrowly distributed Bcc RGP showed classical features of GIs such as a tRNA gene (GI11) or integrase CDS (GI10, GI41). GI10 harboured six IS elements, four from the IS5 and IS66 families and two from the highly prevalent IS3 family (Table 3 ) including the IS407-c element (inactivating a CDS). GI11 contained three elements of the IS3 family and one element of the IS21 family. IS407-e and ISBcen15-b disrupted the same CDS, found to encode a putative helicase (BCAL1308). Another helicase gene (BCAL2480), found in J2315 GI20, was also disrupted by an IS (ISBcen13-b). This could be indicative of a certain level of convergence towards inactivation of such CDSs, but this issue will require further investigation. GI20 showed the highest number of ISs with elements belonging to IS3, IS5, IS21, IS 110 and IS256. The presence of these GIs in the B\&B clone was investigated by PCR screenings and DNA hybridizations. These screenings indicated that GI10 and GI11 together with GI20 and its respective IS407-g element were not present in the $\mathrm{B} \& \mathrm{~B}$ genome or found in the same genomic area. The IS407-g of GI20 was not (a)

RGP with a narrow distribution size $=847.57 \mathrm{~kb}(10.51 \%$ of the genome $)$ IS content $=43$ elements

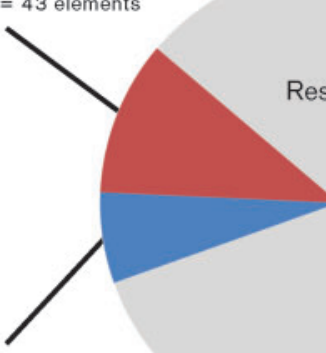

RGP with a broad distribution size $=497.01 \mathrm{~kb}(6.16 \%$ of the genome $)$ IS content $=3$ elements

(c)

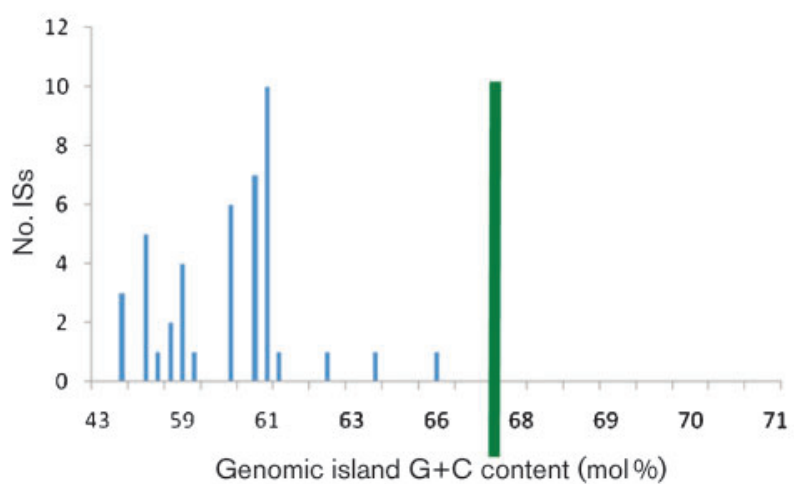

(b)

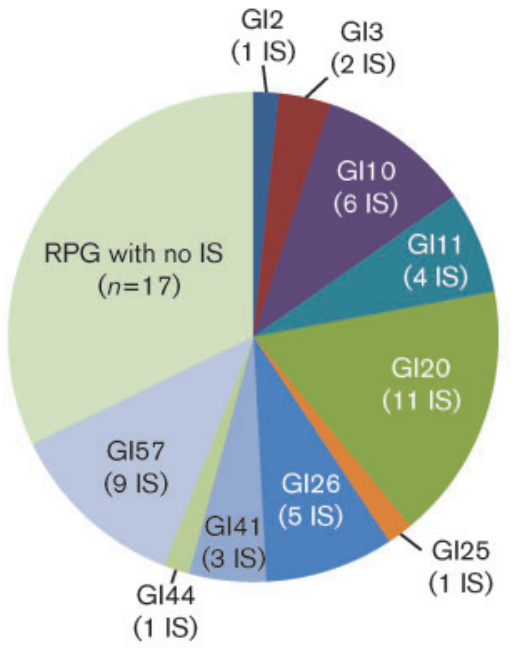

(d)

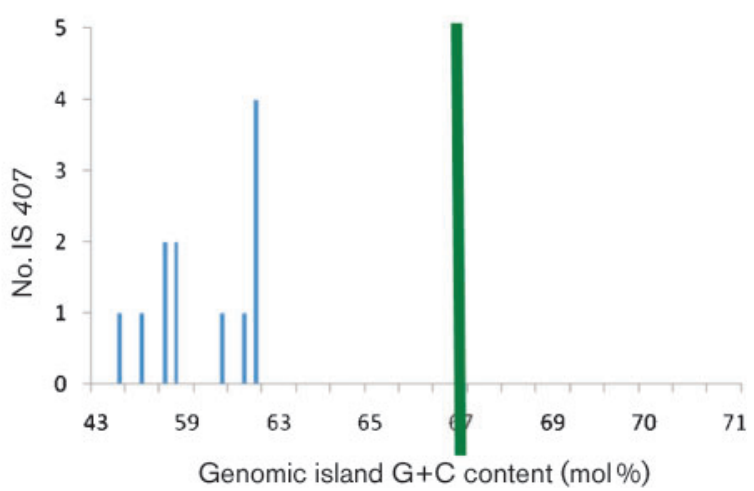

Fig. 4. Illustration of the associations observed between ISs and RGP including Gls. (a) The relative distribution of RGP in $B$. cenocepacia J2315. Narrowly and broadly distributed RGP among the Bcc are given as percentages and the total size of all these RGP is indicated. The number of IS elements per RGP category is shown. (b) IS numbers per RGP and/or GI having a narrow Bcc distribution. (c, d) Plots of the number of ISs (c) and IS 407 (d) against GI G + C content (mol\%) of B. cenocepacia strain J2315 RGP. Data are from Table 3 and Supplementary Table S4. The vertical green bar represents the mean $G+C$ content $(66.9 \mathrm{~mol} \%)$ of the $\mathrm{J} 2315$ full genome. 
detected among all other non-ET12 B. cenocepacia strains tested (Table 2). On GI26, five IS elements from IS3, IS256 and IS66 families were detected (Table 3). The distribution of GI26 among B. cenocepacia was found to be heterogeneous; ET12 lineage strains showed conservation of the entire GI26 RGP but other strains of the recA IIIA PCR RFLP group showed partial GI26 sequences (Parsons et al., 2003) with the exception of the B\&B clone, which contained genetic markers indicative of a GI26 located in the same genomic site as that of J2315. GI41 was defined as a prophage by Holden et al. (2009). Two IS elements of the IS5 family and IS3 families were detected on this RGP (Table 3). ISBcen20-i (IS5) inactivated a putative integrase CDS, probably making this prophage non-lytic. This RGP was not detected by PCR screenings targeting its ends in the closely related $\mathrm{B} \& \mathrm{~B}$ clone. However, the $\mathrm{B} \& \mathrm{~B}$ clone was found to harbour the IS407-l insertion (Table 2). This latter clone is thus likely to harbour this phage or RGP in a distinct genomic region. GI57 showed nine IS elements from the IS3, IS110 and IS21 families (Table 3), with most being on the left end of the GI. Two ISs from GI57 were found to belong to families that have not been recorded so far (ISBcen-unk-b: BCAS0658-0660, and ISBcen-unk-c: BCAS0679A-0681) (Table 1, Fig. 2). A putative partial group II intron, belonging to class E (BCAS0657), was identified close to the zone showing multiple IS elements on GI57. ISBcen2-a was found inserted into a putative integrase CDS of this RGP, probably making it nonmobile. The B\&B genome was found to contain this RGP and its related IS407-m insertion but the latter was in a rearranged form (see above).

\section{Epidemic clones and IS-tagged RGP}

From the above datasets, some ISs appeared to be indicators of narrowly distributed Bcc RGP and GIs. This hypothesis was tested by screening a B\&B clone genomic DNA library using IS407 as a DNA probe. DNA blot analyses of 192 library clones led to the identification of five IS407-positive library clones. Two of these clones were randomly picked for further analysis (clones $5 \mathrm{H} 3=\mathrm{GI}-\mathrm{a}$ and $1 \mathrm{C} 7=\mathrm{GI}-\mathrm{b}$ ). DNA inserts of these clones were partially sequenced and annotated (Fig. 5; GenBank accession nos HQ438284 for GI-a and HQ438285 for GI-b). No significant identities were detected between these RGP and the J2315 sequenced genome. The $\mathrm{G}+\mathrm{C}$ content of the sequenced DNA segments of the B\&B clone GI-a and GI-b were 63.57 and $61.74 \mathrm{~mol} \%$, respectively. BLASTX analysis of the GI-a and GI-b sequences showed similarities with genomic DNA regions of Cupriavidus metallidurans CH34 (GI-a) and Pseudomonas aeruginosa PA14 (GI-b) (Fig. 5). GI-a was found to encode putative proteins that showed strong

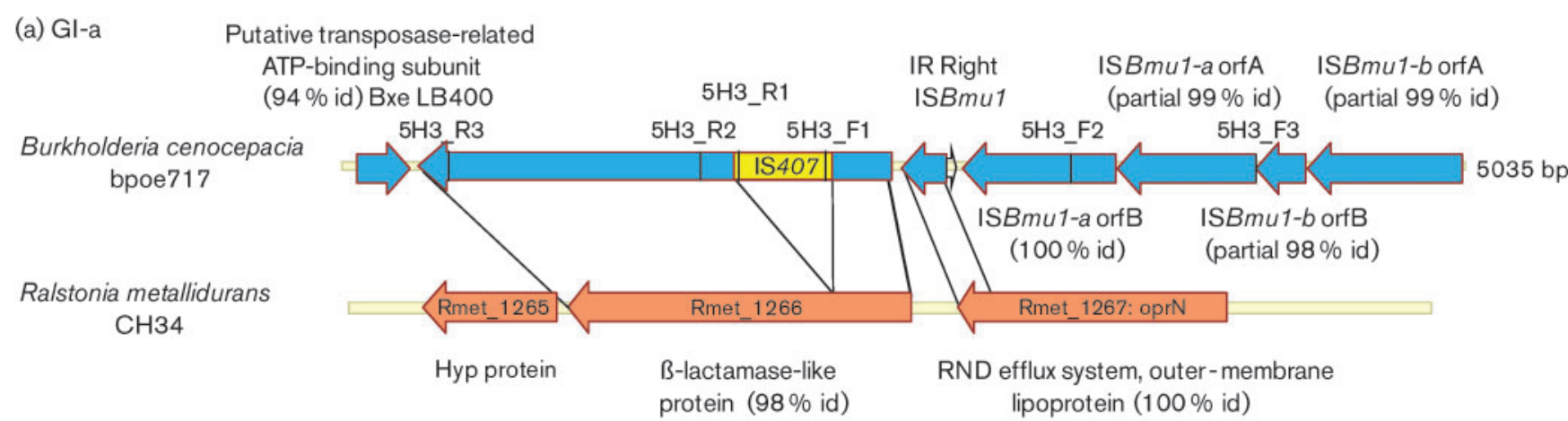

(b) GI-b

Burkholderia cenocepacia bpoe717

Pseudomonas aeruginosa PA14

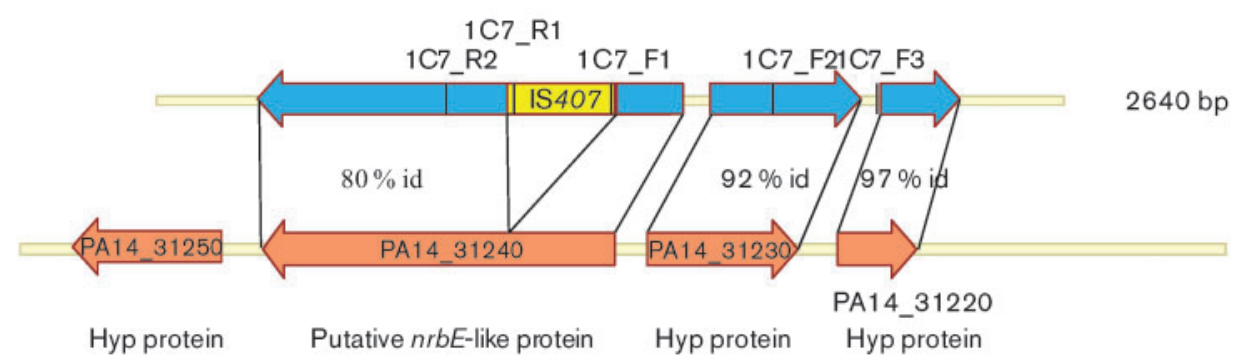

Fig. 5. Genetic structure of recently acquired IS407-tagged Gls of the B\&B clone. Red arrows represent CDSs of the Gl-a (a) and Gl-b (b) sequenced DNA segments. Their respective inferred functions are indicated. Other arrows indicate CDSs that are likely to be found on these islands according to closely related Gls of C. metallidurans for Gl-a and of $P$. aeruginosa for Gl-b. Annotations were inferred from BLAST analyses. The percentage DNA identity (id) with the closest GenBank entry is indicated in parentheses. The primers used for DNA sequencing are given in Supplementary Table S1. 
similarities with a $\beta$-lactamase-like (Rmet_1266) protein and an outer-membrane protein involved in an efflux system (Rmet_1267). PCR screening of the Rmet_1266 gene in the $\mathrm{B} \& \mathrm{~B}$ genomic DNA confirmed this result and showed that this gene was absent from strain J2315. This PCR screening also confirmed the insertion of IS407 in the Rmet_1266 B\&B homologous region. Interestingly, the neighbouring gene, Rmet_1267-homologous CDS, was found to be disrupted by ISBmu1 elements, confirming counterselection of these recently acquired genes. Overall, synteny was conserved between C. metallidurans and clone B\&B sequenced GI-a DNA segments, and high DNA identities (94-100\%) were computed. Comparative genomic analyses showed that the neighbouring C. metallidurans genomic region contained IS21 (Rmet_1263-64) and IS1090 (Rmet_1262) elements, a virD2 type IV secretion system-related gene (Rmet_1259), a traF gene (Rmet_1258) and several features typical of an ICE. The presence of virD2 in the $\mathrm{B} \& \mathrm{~B}$ clone was confirmed by PCR screening and DNA sequencing (data not shown). This $C$. metallidurans genomic region was referred to previously as CMGI-2 (C. metallidurans GI-2), a $101 \mathrm{~kb}$ GI containing at least 25 genes involved in hydrogenotrophy and the metabolism of aromatic compounds (Janssen et al., 2010; Mijnendonckx et al., 2011).

The $\mathrm{B} \& \mathrm{~B}$ clone GI-b was also considered to belong to the category of narrowly distributed Bcc GIs or RGP. The sequenced region of GI-b showed significant identities with the CDS encoding hypothetical proteins (PA14_31230 and PA14_31220) and a partial CDS encoding a deduced NrbE-like protein (BCAL31240) of P. aeruginosa PA14 (Fig. 5b). This latter protein has a domain matching that of a major facilitator protein superfamily, which is involved in multidrug transport (Fluman \& Bibi, 2009). P. aeruginosa PA14 and the B\&B clone showed the same synteny for these three genes, with the exception of IS407, which was not detected in PA14. The Pseudomonas Genome Database (http://www. pseudomonas.com/) showed the corresponding genomic region of PA14 to match a segment with no significant identity with other Pseudomonas genomes. This PA14 region showed a CDS encoding a putative integrase (PA14_31280), a trademark of mobile GIs.

\section{DISCUSSION}

Our views of bacterial species and epidemic clones have changed dramatically over the last decade through the analysis of fully sequenced genomes. Bacterial genomes have been found to be much more plastic than was first thought with, in some instances, massive amounts of DNA having been acquired through horizontal gene transfer events (Wozniak \& Waldor, 2010). Transposable elements such as ISs are peculiar components of these genomes that can inactivate genes or operons and favour recombination events leading to DNA rearrangements and deletions (Drevinek et al., 2010; Miché et al., 2001; Nierman et al., 2004). The benefits of these IS-driven rearrangements can be high and at the heart of the emergence of novel lineages as reported for B. mallei (Song et al., 2010). Nevertheless, their deleterious effects due to their propensity to increase in number, move and favour recombination seem to lead to their gradual loss from bacterial genomes (Wagner, 2006).

The distribution of IS elements among the B. cenocepacia strains investigated in this study indicated a high prevalence (detected among all sequenced genome) of three IS families, IS3, IS110 and IS256, and the probable recent acquisition of some others, such as IS5 and IS66, as inferred from their restricted distribution between closely related strains, the low genetic diversity between copies found in the same genome and their positioning on GIs. Whilst it is obvious that our view of the prevalence of these IS families and subgroups is highly dependent on DNA sequence databases and the availability of full-genome sequences, it is noteworthy that the sequenced J2315 genome has been found, so far, to harbour the highest number of IS elements and families among B. cenocepacia. This strain was thus used as a reference throughout this study. This strain belongs to the ET12 clonal complex and was found to be a close relative of the $\mathrm{B} \& \mathrm{~B}$ clone (Graindorge et al., 2010). IS DNA hybridizations of genomic DNA blots of strain bpoe717 clearly indicated a B\&B IS content similar to that of the J2315 genome, with at least 12 IS3 elements, at least eight IS5 elements and 10 IS110 elements. A high copy number of IS 3 elements was previously considered to be a trademark of the J2315 sequenced genome (Tyler et al., 1996). A similar number in the $\mathrm{B} \& \mathrm{~B}$ clone thus supports the hypothesis of common IS evolutionary trends among the ET12 and B\&B clones. However, Drevinek et al. (2010) did not observe the IS407 and ISBcen 9 subgroups of IS 3 elements among Czech isolates of the ST32 clonal complex, of which the B\&B clone is also a member. This suggests major genetic changes among the ST32 clonal complex.

Genomic localization of IS elements among the $B$. cenocepacia genomes suggests an involvement of IS elements in gene inactivation processes leading to pseudogenes and genetic decay. Global IS distribution did not seem to match particular DNA regions with the exception of a higher occurrence among the CDSs of other IS elements creating IS hotspots or islets. This phenomenon should lead to a gradual loss of the inactivated IS subgroups. It is noteworthy that some IS elements and DNA fragments of various origin were found to have inactivated the same CDS of the $B$. cenocepacia genomes analysed. These inactivations are indicative of convergent evolution and of strong selective pressures favouring the disruption of the same DNA site. However, conserved ISs were rarely observed among a large panel of Bcc strains. In fact, a single IS element, ISBcen8, was found to be conserved at the same site between two sequenced genomes, J2315 and PC184. IS genomic instability thus appeared to be high in B. cenocepacia.

IS407 evolutionary dynamics among B. cenocepacia confirmed this trend. IS407 PCR screening and PCR sequencing 
showed that strains from closely related clonal complexes had the most similar genomic repartitions. The high occurrence of the inactive IS407-i element of the phylogenetic ' $b$ ' cluster of this subgroup among B. cenocepacia suggested this element to be more ancestral than IS407-h. IS407-h probably emerged on GI20 through a duplication of IS407-i, as both elements shared the same ORFB mutation making them pseudo-ISs. With regard to the potentially active IS407 elements of cluster ' $a$ ', IS407-n was considered to be the likely 'founder' element of this cluster, as it is the only cluster ' $a$ ' element found among all $B$. cenocepacia strains that harbours the IS407 subgroup. Moreover, this element was found to be plasmid-borne, and transfer of this plasmid could explain its presence among strains of distinct PCR RFLP subgroups. Analysis of the B\&B clone IS407 loci $(-\mathrm{h},-\mathrm{i}, \mathrm{k}-,-\mathrm{l},-\mathrm{m}$ and $-\mathrm{n})$ revealed highly conserved elements (IS407-l and IS407-n) with those of the 'a' cluster of the J2315 genome but also some elements showing signs of genetic drift (IS407-h, -i and -m) or having been acquired by a convergent insertional event (e. g. IS407-m being in the opposite orientation). Interestingly, IS407-j of J2315 was found to be physically close to the inactive IS407-i and -h elements, suggesting that some DNA regions could favour insertion of such elements. Two other chromosomal areas of more than one element were observed, with one including IS407-a and -b, and the other IS407-d and -e.

Globally, screening performed on 13 strains of B. cenocepacia showed some heterogeneity in IS407 colonization/expansion but confirmed the genomic proximity between the ET12 and $\mathrm{B} \& \mathrm{~B}$ clones. The phenomena at the origin of the differences observed among $B$. cenocepacia strains were partly explained by genome-wide comparisons. These comparisons identified 58 J2315 RGP DNA regions, which sometimes matched the full definition of GIs that were not detected among the B. cenocepacia PHDC genomes. Among these, 27 were considered to be 'narrowly distributed' because of the lack of homology with the other Bcc sequenced genomes. The other RGP were considered to be 'broadly distributed' because of their full or partial detection among other Bcc. Our data showed most Bcc broadly distributed RGP to have no IS element, whilst Bcc narrowly distributed RGP showed from 0 to 10 ISs, with most having at least one IS. This is in line with the idea that DNA regions of low benefit are inactivated to avoid their deleterious effects. In the long run, only the beneficial RGP or GIs (the broadly distributed ones) would thus remain free of pseudogenes, as suggested from the J2315 genomic analysis performed here. IS-tagged RGP would be in a transient state and under selective pressure, which could lead to their rearrangement or loss. However, such RGP/GIs can apparently lead to the introduction of novel IS elements among their recipient hosts. The close association between ISs and RGP/GIs inferred in this study led to the use of IS 407 as a tracer of Bcc narrowly distributed GIs. This IS was successfully used to detect recently acquired GIs in the B. cenocepacia $\mathrm{B} \& \mathrm{~B}$ clone.

IS407 allowed detection of two GIs, GI-a and -b, that were found to be specific for the B\&B clone. These GIs showed significant identity with regions of the $C$. metallidurans $\mathrm{CH} 34$ and $P$. aeruginosa PA14 genomes, respectively. GI-a showed several features of ICEs and harboured a CDS encoding a $\beta$ lactamase-like protein, which was inactivated by an IS407 insertion in the $\mathrm{B} \& \mathrm{~B}$ clone. Surprisingly, this led to the inactivation of a CDS, which could represent a functional benefit for the $\mathrm{B} \& \mathrm{~B}$ clone. However, this clone was previously reported to be resistant to several antibiotics including $\beta$ lactams (Graindorge et al., 2010), thus making this CDS of low added fitness value. B\&B GI-b, also considered a narrowly distributed GI among the Bcc, showed significant identity with a CDS encoding an NrbE-like protein (BCAL31240) in the P. aeruginosa PA14 genome. The encoded protein was found to contain a major facilitator protein superfamily domain, which could be involved in multidrug transport (Fluman \& Bibi, 2009). As with GI-a, the IS407 insertion was not detected in the matching genomic regions of the database. This observation supports the idea of recent insertional events that would have occurred after acquisition by the B\&B clone of these GIs. The Pseudomonas Genome Database showed that the corresponding genomic region of PA14 matched a segment with no significant identity with other Pseudomonas genomes but containing a CDS encoding a putative integrase (PA14_31280), a trademark of mobile GIs. The two B\&B GIs detected by IS407 screening appeared to be recent acquisitions, suggesting an important ability of this clone to acquire foreign DNA.

Overall, this study showed that, even though the B\&B clone is considered part of the ST32 sequence type, its genomic architecture has several features in common with those of the ET12 lineage. In fact, it was found to have several IS407 imprints in line with those of the J2315 (ET12) genome, whilst IS407 was not observed among the ST32 Czech epidemic clone. These data suggest that such clones are highly competent in terms of the acquisition of foreign DNA and that important genomic changes can occur among a $B$. cenocepacia clonal complex. These changes could lead to major functional modifications, which could make these clones better adapted to their recently colonized habitat and play a key part in their epidemic spread.

\section{ACKNOWLEDGEMENTS}

A. G. was supported by a PhD fellowship from the 'Cluster Environnement' of the Rhône-Alpes Region (France) and by Université Lyon 1. We thank the CNRS, Université Lyon 1, and Rhône-Alpes Region (France) for having funded parts of this work. This work was also partly funded by the Agence Nationale de la Recherche (ANR) SEST 2005009 and CESA 2008022 projects, and the 'Association Vaincre la Mucoviscidose'. We thank H. De Montclos and F. Laurent of the Fleyriat Hospital of Bourg-en-Bresse, and S. FavreBonté of University Lyon 1(France) for their comments on this work.

\section{REFERENCES}

Abalain, M. L., Héry-Arnaud, G., Gouriou, S., Lintanf, J., Barrere, V., Segonds, C., Chabanon, G., Rault, G., Barbier, G. \& Payan, C. (2009). Clonally identical Burkholderia cepacia complex (Bcc) sequence type 
strain ST32 isolated from cystic fibrosis (CF) patients in French western Brittany compared to epidemiology of Bcc strains in France using multilocus sequence typing (MLST). J Cyst Fibros 8, S34.

Bauer, D. W. \& Collmer, A. (1997). Molecular cloning, characterization, and mutagenesis of a pel gene from Pseudomonas syringae pv. lachrymans encoding a member of the Erwinia chrysanthemi pelADE family of pectate lyases. Mol Plant Microbe Interact 10, 369-379.

Bertani, G. (1951). Studies on lysogenesis. I. The mode of phage liberation by lysogenic Escherichia coli. J Bacteriol 62, 293-300.

Carver, T. J., Rutherford, K. M., Berriman, M., Rajandream, M. A., Barrell, B. G. \& Parkhill, J. (2005). АСТ: the Artemis Comparison Tool. Bioinformatics 21, 3422-3423.

Coenye, T. \& LiPuma, J. J. (2003). Population structure analysis of Burkholderia cepacia genomovar III: varying degrees of genetic recombination characterize major clonal complexes. Microbiology 149, 77-88

Coenye, T., Spilker, T., Van Schoor, A., LiPuma, J. J. \& Vandamme, P. (2004). Recovery of Burkholderia cenocepacia strain PHDC from cystic fibrosis patients in Europe. Thorax 59, 952-954.

Cournoyer, B. \& Normand, P. (1994). Characterization of a spontaneous thiostrepton-resistant Frankia alni infective isolate using PCRRFLP of nif and glnII genes. Soil Biol Biochem 26, 553-559.

Drevinek, P. \& Mahenthiralingam, E. (2010). Burkholderia cenocepacia in cystic fibrosis: epidemiology and molecular mechanisms of virulence. Clin Microbiol Infect 16, 821-830.

Drevinek, P., Baldwin, A., Lindenburg, L., Joshi, L. T., Marchbank, A., Vosahlikova, S., Dowson, C. G. \& Mahenthiralingam, E. (2010). Oxidative stress of Burkholderia cenocepacia induces insertion sequencemediated genomic rearrangements that interfere with macrorestrictionbased genotyping. J Clin Microbiol 48, 34-40.

Fluman, N. \& Bibi, E. (2009). Bacterial multidrug transport through the lens of the major facilitator superfamily. Biochim Biophys Acta 1794, 738-747.

Godoy, D., Randle, G., Simpson, A. J., Aanensen, D. M., Pitt, T. L., Kinoshita, R. \& Spratt, B. G. (2003). Multilocus sequence typing and evolutionary relationships among the causative agents of melioidosis and glanders, Burkholderia pseudomallei and Burkholderia mallei. J Clin Microbiol 41, 2068-2079.

Graindorge, A., Menard, A., Neto, M., Bouvet, C., Miollan, R., Gaillard, S. de Montclos, H., Laurent, F. \& Cournoyer, B. (2010). Epidemiology and molecular characterization of a clone of Burkholderia cenocepacia responsible for nosocomial pulmonary tract infections in a French intensive care unit. Diagn Microbiol Infect Dis 66, 29-40.

Holden, M. T., Seth-Smith, H. M., Crossman, L. C., Sebaihia, M., Bentley, S. D., Cerdeño-Tárraga, A. M., Thomson, N. R., Bason, N., Quail, M. A. \& other authors (2009). The genome of Burkholderia cenocepacia J2315, an epidemic pathogen of cystic fibrosis patients. J Bacteriol 191, 261-277.

Isles, A., Maclusky, I., Corey, M., Gold, R., Prober, C., Fleming, P. \& Levison, H. (1984). Pseudomonas cepacia infection in cystic fibrosis: an emerging problem. J Pediatr 104, 206-210.

Janssen, P. J., Van Houdt, R., Moors, H., Monsieurs, P., Morin, N., Michaux, A., Benotmane, M. A., Leys, N., Vallaeys, T. \& other authors (2010). The complete genome sequence of Cupriavidus metallidurans strain $\mathrm{CH} 34$, a master survivalist in harsh and anthropogenic environments. PLOS ONE 5, e10433.

Johnson, W. M., Tyler, S. D. \& Rozee, K. R. (1994). Linkage analysis of geographic and clinical clusters in Pseudomonas cepacia infections by multilocus enzyme electrophoresis and ribotyping. J Clin Microbiol 32, 924-930.

Kenna, D. T., Yesilkaya, H., Forbes, K. J., Barcus, V. A., Vandamme, P. \& Govan, J. R. (2006). Distribution and genomic location of active insertion sequences in the Burkholderia cepacia complex. J Med Microbiol 55, 1-10.

Kumar, S., Tamura, K. \& Nei, M. (2004). MEGA3: integrated software for molecular evolutionary genetics analysis and sequence alignment. Brief Bioinform 5, 150-163.

LiPuma, J. J., Mortensen, J. E., Dasen, S. E., Edlind, T. D., Schidlow, D. V., Burns, J. L. \& Stull, T. L. (1988). Ribotype analysis of Pseudomonas cepacia from cystic fibrosis treatment centers. J Pediatr 113, 859-862.

Liu, L., Spilker, T., Coenye, T. \& LiPuma, J. J. (2003). Identification by subtractive hybridization of a novel insertion element specific for two widespread Burkholderia cepacia genomovar III strains. J Clin Microbiol 41, 2471-2476.

Mahenthiralingam, E., Bischof, J., Byrne, S. K., Radomski, C., Davies, J. E., Av-Gay, Y. \& Vandamme, P. (2000). DNA-based diagnostic approaches for identification of Burkholderia cepacia complex, Burkholderia vietnamiensis, Burkholderia multivorans, Burkholderia stabilis, and Burkholderia cepacia genomovars I and III. J Clin Microbiol 38, 3165-3173.

Mahillon, J. \& Chandler, M. (1998). Insertion sequences. Microbiol Mol Biol Rev 62, 725-774.

Menard, A., Monnez, C., Estrada de Los Santos, P., Segonds, C., Caballero-Mellado, J., Lipuma, J. J., Chabanon, G. \& Cournoyer, B. (2007). Selection of nitrogen-fixing deficient Burkholderia vietnamiensis strains by cystic fibrosis patients: involvement of nif gene deletions and auxotrophic mutations. Environ Microbiol 9, 11761185.

Miché, L., Faure, D., Blot, M., Cabanne-Giuli, E. \& Balandreau, J. (2001). Detection and activity of insertion sequences in environmental strains of Burkholderia. Environ Microbiol 3, 766-773.

Mijnendonckx, K., Provoost, A., Monsieurs, P., Leys, N., Mergeay, M., Mahillon, J. \& Van Houdt, R. (2011). Insertion sequence elements in Cupriavidus metallidurans $\mathrm{CH} 34$ : distribution and role in adaptation. Plasmid 65, 193-203.

Nierman, W. C., DeShazer, D., Kim, H. S., Tettelin, H., Nelson, K. E., Feldblyum, T., Ulrich, R. L., Ronning, C. M., Brinkac, L. M. \& other authors (2004). Structural flexibility in the Burkholderia mallei genome. Proc Natl Acad Sci U S A 101, 14246-14251.

Parsons, Y. N., Banasko, R., Detsika, M. G., Duangsonk, K., Rainbow, L., Hart, C. A. \& Winstanley, C. (2003). Suppression-subtractive hybridisation reveals variations in gene distribution amongst the Burkholderia cepacia complex, including the presence in some strains of a genomic island containing putative polysaccharide production genes. Arch Microbiol 179, 214-223.

Pitcher, D. G., Saunders, N. A. \& Owen, R. J. (1989). Rapid extraction of bacterial genomic DNA with guanidium thiocyanate. Lett Appl Microbiol 8, 151-156.

Saiman, L. \& Siegel, J. (2004). Infection control in cystic fibrosis. Clin Microbiol Rev 17, 57-71.

Saitou, N. \& Nei, M. (1987). The neighbor-joining method: a new method for reconstructing phylogenetic trees. Mol Biol Evol 4, 406425.

Sambrook, J., Fritsch, E. F. \& Maniatis, T. (1989). Molecular Cloning: a Laboratory Manual. Cold Spring Harbor, NY: Cold Spring Harbor Laboratory Press.

Song, H., Hwang, J., Yi, H., Ulrich, R. L., Yu, Y., Nierman, W. C. \& Kim, H. S. (2010). The early stage of bacterial genome-reductive evolution in the host. PLoS Pathog 6, e1000922.

Tyler, S. D., Rozee, K. R. \& Johnson, W. M. (1996). Identification of IS1356, a new insertion sequence, and its association with IS402 in epidemic strains of Burkholderia cepacia infecting cystic fibrosis patients. J Clin Microbiol 34, 1610-1616. 
Vanlaere, E., Baldwin, A., Gevers, D., Henry, D., De Brandt, E., LiPuma, J. J., Mahenthiralingam, E., Speert, D. P., Dowson, C. \& Vandamme, P. (2009). Taxon K, a complex within the Burkholderia cepacia complex, comprises at least two novel species, Burkholderia contaminans sp. nov. and Burkholderia lata sp. nov. Int J Syst Evol Microbiol 59, 102-111.

Vial, L., Chapalain, A., Groleau, M. C. \& Déziel, E. (2011). The various lifestyles of the Burkholderia cepacia complex species: a tribute to adaptation. Environ Microbiol 13, 1-12.
Wagner, A. (2006). Periodic extinctions of transposable elements in bacterial lineages: evidence from intragenomic variation in multiple genomes. Mol Biol Evol 23, 723-733.

Wagner, A. (2009). Transposable elements as genomic diseases. Mol Biosyst 5, 32-35.

Wozniak, R. A. \& Waldor, M. K. (2010). Integrative and conjugative elements: mosaic mobile genetic elements enabling dynamic lateral gene flow. Nat Rev Microbiol 8, 552-563. 